Relations industrielles

Industrial Relations

\title{
The Functional Economy, by Bernard William Dempsey, Englewood Cliffs, N.J., Prentice-Hall Inc., 1958, 515 pp.
}

\section{Gérald Dion}

Volume 15, numéro 1, janvier 1960

URI : https://id.erudit.org/iderudit/1022078ar

DOI : https://doi.org/10.7202/1022078ar

Aller au sommaire du numéro

Éditeur(s)

Département des relations industrielles de l’Université Laval

ISSN

0034-379X (imprimé)

1703-8138 (numérique)

Découvrir la revue

Citer ce compte rendu

Dion, G. (1960). Compte rendu de [The Functional Economy, by Bernard William Dempsey, Englewood Cliffs, N.J., Prentice-Hall Inc., 1958, 515 pp.] Relations industrielles / Industrial Relations, 15(1), 141-142.

https://doi.org/10.7202/1022078ar

Tous droits réservés (C Département des relations industrielles de l’Université Laval, 1960
Ce document est protégé par la loi sur le droit d'auteur. L'utilisation des services d'Érudit (y compris la reproduction) est assujettie à sa politique d'utilisation que vous pouvez consulter en ligne.

https://apropos.erudit.org/fr/usagers/politique-dutilisation/ 
volonté de la part des employés de la requérante, et $œ$, plus particulièrement à cause de tous les événements qui s'étaient déroulés.

L'acte qu'elle a prescrit, à savoir, le vote - et ce, en l'absence de toute disposition règlementaire applicable au cas particulier qui lui était soumis - en était un qu'à mon avis elle aurait pu prescrire « par règlement et avec le même effet 》, sans ainsi excéder la juridiction qui lui appartient en vertu des articles 3 à 10 de la Loi des relations ouvrières.

Pour toutes ces raisons, la demande de la requérante pour l'émission d'un bref de prohibition est rejetée, avec dépens.

\section{RECENSIONS - BOOK REVIEWS}

The Functional Economy, by Bernard William Dempsey, Englewood Cliffs, N.J., Prentice-Hall Inc., 1958, 515 pp.

L'auteur est bien connu. Il s'est rendu célèbre par la traduction en anglais et la présentation du commentaire de Quadragesimo Anno qu'avait publié Oswald Von Nell-Breuning, Reorganization of Social Economy (Bruce, 1936).

L'idée de base qui constitue l'unité de The Functional Economy est celle qu'il a prise dans l'enseignement de Pie XI sur la création de groupements intermédiaires entre les individus et l'Etat chargés de la gérance du bien commun au sein de la profession. Elle revient dans tous ces chapitres, plus ou moins rattachés les uns aux autres, qui forment son ouvrage. On y trouve de tout, de l'histoire sociale, de la théorie ́́conomique, de la morale sociale, une analyse de la situation économico-sociale américaine.

Pour l'auteur, il n'existe aucun doute que toute l'économie américaine repose sur une inconsciente conception de lutte de classe. Il y fait souvent allusion. Nous prenons une citation au hasard. "The acceptance of class conflict not merely as a fact, but as a valid principle has been, if not actually universal in American industrial society, at least very common indeed. This one erroneous idea, common to both liberalism and socialism, has been accepted as a settled and established point of departure in dispute by labor and business alike, and by the Congress and the Courts which viewed the industrial world in which these factions live, interpreted it, legislated for it, and made decisions concerning it 》. (p. 144)

Nous sommes loin d'être certains que cette hypothèse explicative du phénomène des relations du travail aux Etats-Unis soit juste. En tous cas, elle est très contestée. D'abord, jusqu'à quel point $y$-a-t-il des classes aux Etats-Unis? Peut-on parler de classe de capitalistes et de prolétaires en mettant d'un côté ceux qui gouvernent l'industrie et de lautre les travailleurs? Les sociologues ont montré qu'il existe une conscience collective très faible chez les travailleurs, que ceux-ci avaient une double loyauté envers leur union et envers l'entreprise où ils travaillent. $S^{\prime}$ il est vrai que du côté de la direction des entreprises et surtout du côté des Associations d'employeurs il existe vraiment un esprit belligérant contre les syndicats ouvriers (non contre les travailleurs), malgré les textes tirés des anciennes constitutions de l'AFL ou de certaines unions qui lui étaient affiliées. ni les syndicats ouvriers ni les travailleurs n'entretiennent à l'endroit des capitalistes un esprit de lutte de classe. Il n'y a pas de conflits de classe, mais conflits d'intérêts entre organismes représentants les agents de production.

Nous croyons que l'auteur a été trop influencé par les analyses de la situation européenne et qu'il n'a pas suf- 
fisamment pris la peine de considérer attentivement le phénomène américain en utilisant des catégories qui pouvaient l'expliquer tel qu'il est. Il n'est d'ailleurs pas le seul à verser dans cette déformation.

Ces réserves, si importantes qu'elles soient, ne diminuent pas les mérites de certaines parties de l'ouvrage, en particulier de celles qui concernent les principes de la propriété privée, les bases d'un juste salaire, et le développement d'une organisation fonctionnelle.

\section{Gérard Dion}

\section{Human Relations in Administration,} by R. Dubin, Englewood Cliffs, N.J., Prentice-Hall, 1957.

Quand un lecteur aborde un volume dont le titre inclut les mots de relations humaines, il s'attend d'y trouver des enquêtes et entrevues sur le moral et les attitudes des employés, des statistiques sur l'âge, l'éducation, l'ancienneté, le roulement de la main-d'oeuvre, l'absentéisme, etc... et enfin des corrélations entre diverses mesures de satisfaction et de rendement. Cette méthode d'analyse s'appuie sur l'étude d'un seul segment de toute société ou organisation industrielle: l'employé. En général, elle ignore la structure même de l'organisation, les attributs qui la caractérisent et les individus qui l'animent: la gérance, les spécialistes et l'employé.

Justement ce volume de Dubin est probablement l'un des, premiers qui s'attaquent à l'étude d'une organisation industrielle en considérant sa structure et ses qualités distinctives. Nous ne sommes pas d'accord avec Dubin quand il affirme que cet ouvrage est basé sur la recherche. Il rapporte surtout les considérations et les expériences personnelles de chaque individu qui a collaboré à la préparation du volume. Ces collaborateurs sont surtout des sociologues.

Le présent ouvrage n'est pas récent puisqu'il fut imprimé d'abord pour la première fois en 1951 et que les références sont antérieures à cette année-là.

La première partie du volume se compose de 21 chapitres. Chacun d'eux comprend plusieurs articles rédigés par des auteurs différents. Comme le style et l'école sociologique varient d'un auteur à l'autre, la lecture du volume est ardue et plutôt difficile. En effet, le lecteur n'a pas le temps de s'adapter aux différents styles.

L'auteur décrit en premier lieu l'organisation en tant que système social et ensuite il considère les motivations évoquées par ce système. Les relations qui s'établissent alors entre les membres de cette organisation découlent des procédures inhérentes à la structure elle-même. La structure des différents systèmes administratifs fait l'objet de plusieurs chapitres, de même que les attributs des membres. Les articles sur les « exécutifs », les spécialistes et les contremaîtres pénètrent si bien les dynamiques en jeu que le lecteur acquiert un bon aperçu de toute la situation. Prendre des décisions, i.e. 《decision-making s est-il un art ou une science? Ici il n'est pas question de la science des décisions (Operations Research), mais de la variété des décisions et des limites imposées à la gérance par la structure de porganisation.

La deuxième partie du volume comprend uniquement des cas à discuter. Ces cas se rapportent surtout aux relations entre patrons et employés. $\mathrm{Hu}$ man Relations in Administration * serait surtout utile comme texte de base pour un cours ayant une période théorique suivie d'une période de discussion de cas.

\section{G. R. Graoux}

Compulsory Conciliation and Collective Bargaining, The New Brunswick Experience, by W. B. Cunningham. Published by The New Brunswick Department of Labour, Fredericton, N.B. and The Industrial Relations Centre, McGill University, Montreal, 123 pp.

Depuis quelques années, on assiste à un mouvement de plus en plus répandu d'examen et de critique du système de conciliation obligatoire à travers le Canada. Le présent ouvrage s'ajoute au dossier déjà impressionnant de l'analyse de cette forme d'intervention gouvernementale dans le règlement des conflits du travail. 